\title{
Hybrid Tracking and Mosaicking for Information Augmentation in Retinal Surgery
}

\author{
Rogério Richa, Balázs Vágvölgyi, Marcin Balicki, \\ Gregory D. Hager, and Russell H. Taylor \\ Johns Hopkins University \\ rogerio.richa@jhu.edu
}

\begin{abstract}
Current technical limitations in retinal surgery hinder the ability of surgeons to identify and localize surgical targets, increasing operating times and risks of surgical error. In this paper we present a hybrid tracking and mosaicking method for augmented reality in retinal surgery. The system is a combination of direct and feature-based tracking methods. A novel extension for direct visual tracking using a robust image similarity measure in color images is also proposed. Several experiments conducted on phantom, in vivo rabbit and human images attest the ability of the method to cope with the challenging retinal surgery scenario. Applications of the proposed method for tele-mentoring and intra-operative guidance are demonstrated.
\end{abstract}

Keywords: tracking, mosaicking, information augmentation, retinal surgery.

\section{Introduction}

Retinal surgery is considered one of the most demanding types of surgical intervention. Difficulties related to this type of surgery arise from several factors such as the difficult visualization of surgical targets, poor ergonomics, lack of tactile feedback, complex anatomy and high accuracy requirements. Specifically regarding intra-operative visualization, surgeons face limitations in field and clarity of view, depth perception and illumination which hinder their ability to identify and localize surgical targets. These limitations result in long operating times and risks of surgical error.

A number of solutions for aiding surgeons during retinal surgery including robotic assistants for improving surgical accuracy and mitigating the impact of physiological hand tremor [1], micro-robots for drug delivery [2] and sensing instruments for intra-operative data acquisition [3] have been proposed. Addressing the limitations in visualization, systems for intra-operative view expansion and information overlay have been developed in 45]. In such systems, a mosaic of the retina is created intra-operatively and pre-operative surgical planning and data (e.g. fundus images) are displayed during surgery for improved guidance.

Although several solutions have been proposed in the field of minimally invasive surgery and functional imaging [67], retinal surgery imposes additional 


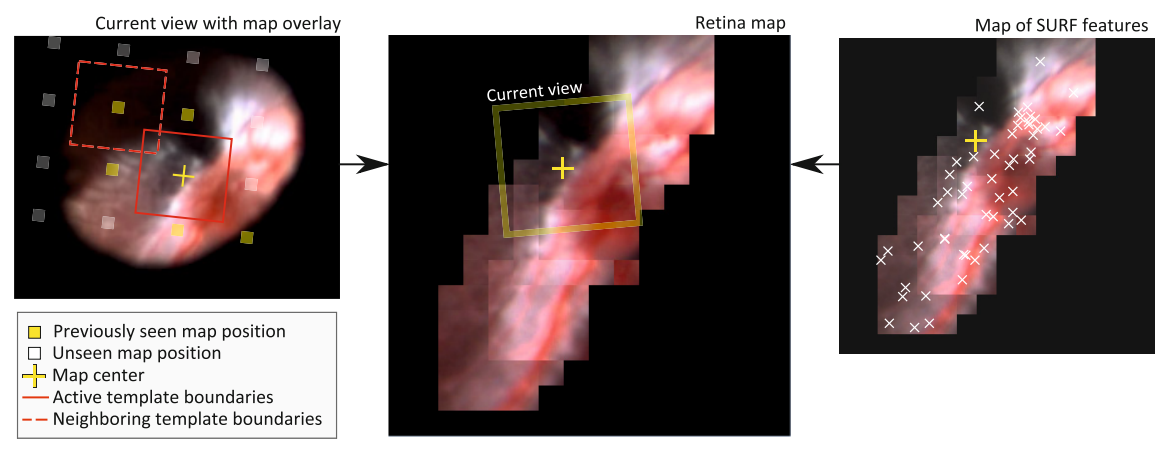

Fig. 1. The proposed hybrid tracking and mosaicking method. A direct visual tracking method (left) is combined with a SURF feature map (right) for coping with full occlusions. The result is the intra-operative retina map shown in the middle. Notice the retina map displayed above is a simple overlay of the templates associated with each map position.

challenges such as highly variable illumination (the illumination source is manually manipulated inside the eye), partial and full occlusions, focus blur due to narrow depth of field and distortions caused by the eye lens. Although the systems proposed in [45] suggest potential improvements in surgical guidance, they lack robustness to such disturbances. Furthermore, tracking and mapping in such conditions is much harder compared to non-invasive retinal interventions such as laser photocoagulation 8 .

In this paper we present a hybrid tracking and mosaicking method designed for the challenging conditions in retinal surgery. The method is a combination of both direct and feature-based tracking methods. Similar to [5] and [9], a two dimensional map of the retina is build on-the-fly using a direct tracking method based on a robust similarity measure called Sum of Conditional Variance (SCV) [10. In parallel, a map of SURF features is built and updated as the map expands, enabling tracking to be reinitialized in case of full occlusions. The method has been tested on a database of phantom, rabbit and human surgeries, with successful results.

This paper is organized as follows. In the next section, we describe the components of the proposed hybrid tracking and mosaicking method. In section 3 . we describe the experimental analysis conducted on in vivo data. In section 4 we demonstrate potential applications of the proposed system in intra-operative navigation and tele-mentoring systems. We conclude the paper in section 5 .

\section{Methods}

A schematic overview of the proposed hybrid tracking and mosaicking method is given in Figure 1. A combination of feature-based and direct based methods was necessary due to the specific nature of the retina images, where low frequency texture information is predominant. As explained in details in section 3 
a purely feature-based tracking and mosaicking method could not produce the same results as the proposed method in the in vivo human datasets shown in Figure 3 due to the lack of salient features in certain areas of the retina.

During surgery, only a small portion of the retina is visible. For initializing the tracking and mosaicking method, an initial reference image of the retina is selected. The center of the initial reference image represents the origin of a retina map. As the surgeon explores the retina, additional templates are incorporated to the map, as the distance to the map origin increases. New templates are recorded at even spaces, as illustrated in Figure 1(left) (notice that regions of adjacent templates overlap). At a given moment, the template closest to the current view of the retina is tracked using the direct tracking method detailed next.

\subsection{Direct Visual Tracking Using a Robust Similarity Measure}

As explained in the introduction, tracking must cope with disturbances such as illumination variations, partial occlusions (e.g. due to particles floating in the vitreous), distortions, etc. To this end, we tested several robust image similarity measures from the medical image registration domain such as Mutual Information (MI), Cross Cumulative Residual Entropy (CCRE), Normalized Cross Correlation (NCC) and the Sum of Conditional Variance (SCV) (see [10] for more information). Among these measures, the SCV has shown the best tradeoff between robustness and convergence radius. In addition, efficient optimization schemes can be derived for the SCV, which is not the case for NCC, MI or CCRE.

Tracking can be formulated as an optimization problem, where we seek to find at every image the parameters $\mathbf{p}$ of the transformation function $w(\mathbf{x}, \mathbf{p})$ that minimize the SCV between the template and current images $T$ and $I(w(\mathbf{x}, \mathbf{p}))$ :

$$
\mathcal{S C V}(\mathbf{p})=\sum_{\mathbf{x}}\left(I(w(\mathbf{x}, \mathbf{p}))-\hat{T}_{(i, j)}(\mathbf{x})\right)^{2}, \text { with } \hat{T}(\mathbf{x})=\mathcal{E}\left(I(w(\mathbf{x}, \mathbf{p})) \mid T_{(i, j)}(\mathbf{x})\right)
$$

where $\mathcal{E}($.$) is the expectation operator. The indexes (i, j)$ represent the row and column of the template position in the retinal map shown in Figure 1 The transformation function $w($.$) is chosen to be a similarity transformation$ (4 DOFs, accounting for scaling, rotation and translation). Notice that more complex models such as the quadratic model [11] can be employed for mapping with higher accuracy.

In the medical imaging domain, images $T$ and $I$ are usually intensity images. Initial tests of retina tracking in gray-scale images yielded poor tracking performance due to the lack of image texture in certain parts of the retina. This motivated the extension of the original formulation in equation (1) to tracking in color images for increased robustness:

$$
\mathcal{S C} \mathcal{V}^{*}(\mathbf{p})=\sum_{\mathbf{c}} \sum_{\mathbf{x}}\left({ }^{c} I(w(\mathbf{x}, \mathbf{p}))-{ }^{c} \hat{T}_{(i, j)}(\mathbf{x})\right)^{2}
$$

In the specific context of retinal images, the blue channel could be ignored as it is not a strong color component. Hence, tracking is performed using red and 


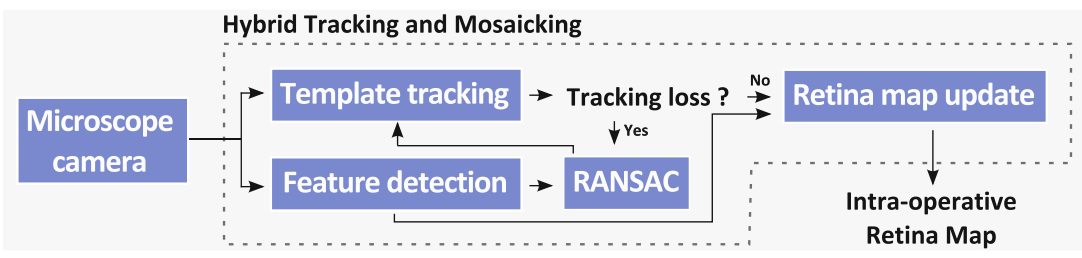

Fig. 2. Schematic overview of the proposed tracking system

green channels. For finding the transformation parameters $\mathbf{p}$ that minimize equation (2), the Efficient Second-Order Minimization (ESM) strategy is adopted [9]. Finally, it is important to highlight the fact that new templates are only incorporated to the retina map when tracking confidence is high (i.e. over an empirically defined threshold $\epsilon$ ). Once a given template is incorporated to the map, it is no longer updated. Tracking confidence is measured as the average NCC between ${ }^{c} \hat{T}$ and ${ }^{c} I(w(\mathbf{x}, \mathbf{p}))$ over red and green color channels $c$. Notice this specific NCC is a bounded measure derived directly from the SCV coefficient (the expected ${ }^{c} \hat{T}$ is used instead of $\left.{ }^{c} T\right)$.

\subsection{Creating a Feature Map}

For recovering tracking in case of full occlusions, a map of SURF features on the retina is also created. For every new template incorporated in the map, the set of SURF features within the new template is included in the feature map. Due to the overlap between templates, new features are matched against the existing features in the map and if the distance (in pixels) between old and new features on the map is small, two features are merged by taking the average of their positions and descriptor vectors.

If the template tracking confidence drops below a pre-defined threshold $\lambda$, tracking is deemed unreliable and is suspended. For re-establishing tracking, RANSAC is employed for matching features from the feature map to those on the current image from the surgery. In practice, due to the poor visualization conditions in retinal surgery, the SURF Hessian thresholds are set very low. This creates a large number of false matches, which consequently requires a large number of RANSAC iterations. A schematic diagram of the hybrid tracking and mosaicking method is shown in Figure 2 ,

\section{Experiments}

For acquiring phantom and in vivo rabbit images, we use a FireWire Point Grey camera acquiring $800 \times 600$ pixel color images at 25 fps. For the in vivo human sequences, a standard NTSC camera acquiring 640x480 color images was used. The method was implemented using OpenCV on a Xeon $2.10 \mathrm{GHz}$ machine. The direct tracking branch (see Figure 2) runs at frame-rate while the feature detection and RANSAC branch runs at $\approx 6$ fps (depending on the 

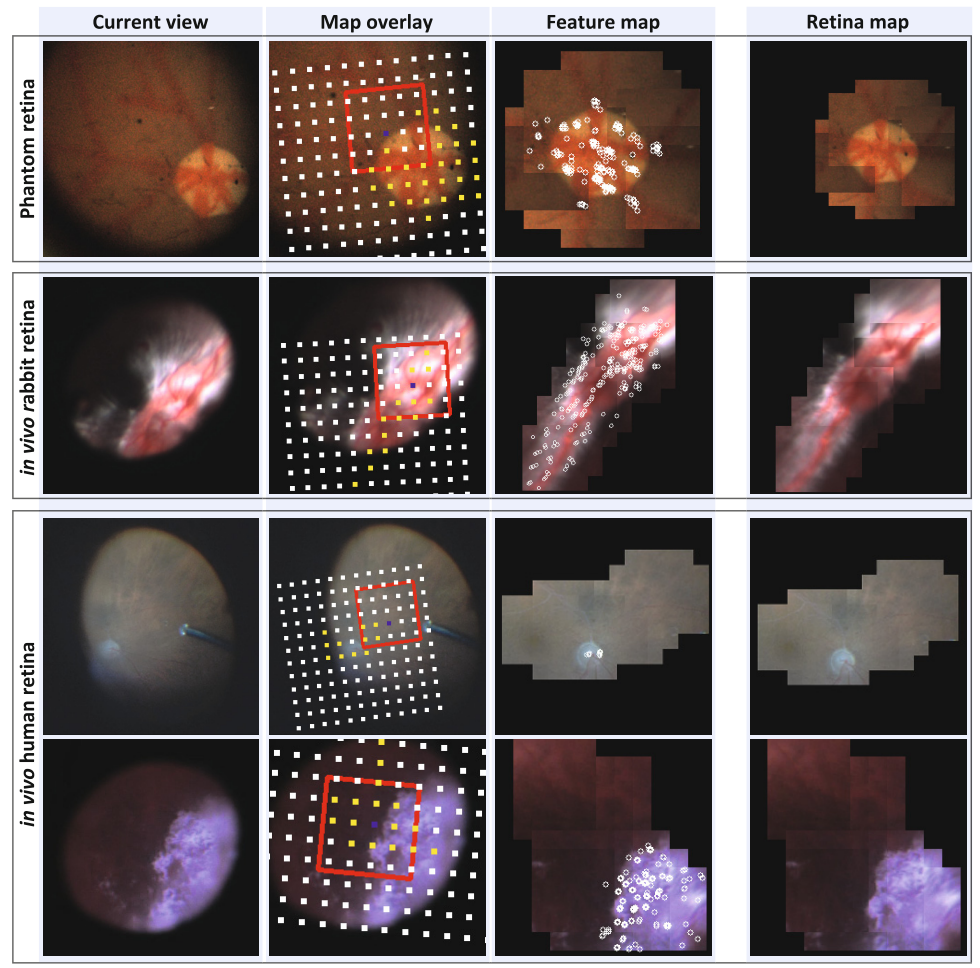

Fig. 3. Examples of intra-operative retina maps obtained using the proposed hybrid tracking and mosaicking method

number of detected features). Although the two branches already run in parallel, considerable speed gains can be achieved with further code optimization.

\subsection{Reconstructed Retina Maps}

Figure 3 shows examples of retina maps obtained using the proposed method. In addition, an example of an on-line map reconstruction can be found in the supplementary videos. For all sequences, we set the template size for each map position to $90 \times 90$ pixels. Map positions are evenly spaced by 30 pixels. Due to differences in the acquisition setup (zoom level, pupil dilation, etc), the field of view may vary between sequences. The rabbit image dataset consists of two sequences of $15 \mathrm{~s}$ and $20 \mathrm{~s}$ and the human image datasets consist of two sequences of $46 \mathrm{~s}$ and 39s (lines 3 and 4 in Figure 3 , respectively). The tracking confidence threshold $\epsilon$ for incorporating new templates and the threshold $\lambda$ for detecting tracking failure were empirically set to 0.95 and 0.6 , respectively, for all experiments. In addition, the number of RANSAC iterations was set to 1000. 

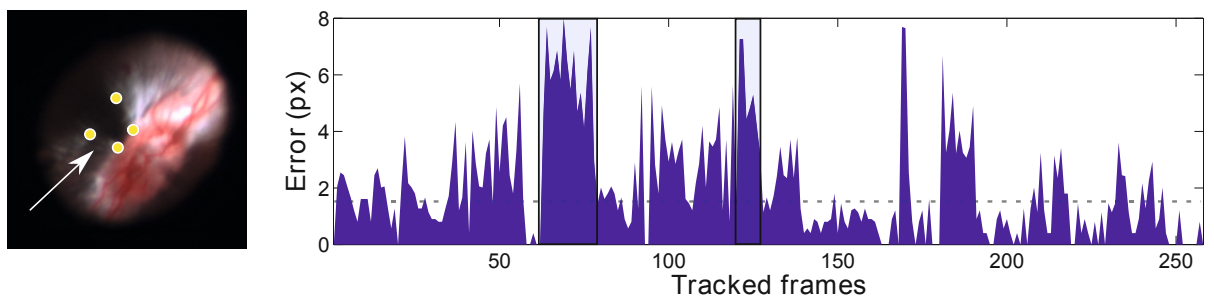

Fig. 4. For a quantitative analysis, the average tracking error of four points arbitrarily chosen on the rabbit retina is manually measured. Slight tracking drifts are highlighted in the plot.

The advantages of the proposed extension to tracking in color are clearly shown in the experiments with human in vivo images. In these specific images, much information is lost in the convertion to gray-scale, reducing the tracking convergence radius and increasing chances of tracking failure. Consequently, the estimated retina map is considerably smaller than when tracking in color images (see example in 5(a)).

For a quantitative analysis of the proposed method, we manually measured the tracking error (in pixels) of four points arbitrarily chosen on 500 images of the rabbit retina shown in Figure 4. The error is only measured when tracking is active (i.e. tracking confidence above $\epsilon$ ). In average, tracking error is below $1.60 \pm 3.1$ pixels, which is close the manual labeling accuracy (estimated to be $\approx 1$ pixel). Using the surgical tool shaft as reference in this specific image sequence, the ratio between pixels and millimeters is approximately $20 \mathrm{px} / \mathrm{mm}$. From the plot, slight tracking drifts can be detected (from frame intervals $[60,74]$ and $[119,129]$ highlighted in the plot), as well as error spikes caused by image distortions. Overall, even though tracking accuracy is too large for applications such as robotic assisted vein cannulation, it is sufficient for consistent video overlay.

\subsection{Current Limitations}

A visual inspection of Figure 3 suggests a correlation between SURF features and blood vessels on the retina. In fact, few features are available where blood vessels are not clearly visible or in regions distant from the nerve bundle. In the proposed method, a minimum number of feature matches is necessary to recover from full occlusions. A possible solution to circumvent this issue is the use of additional feature detection methods adapted to retinal images.

The proposed tracking and mosaicking method strongly relies on the NCC coefficient as a measure of tracking quality. However, the NCC does not provide reliable results when tracking images with little texture information. Since the transformation model is not constrained, tracking errors result in the incorporation of falsetemplates to the map (see Figure $5(\mathrm{~b})$ ). As an alternative to 

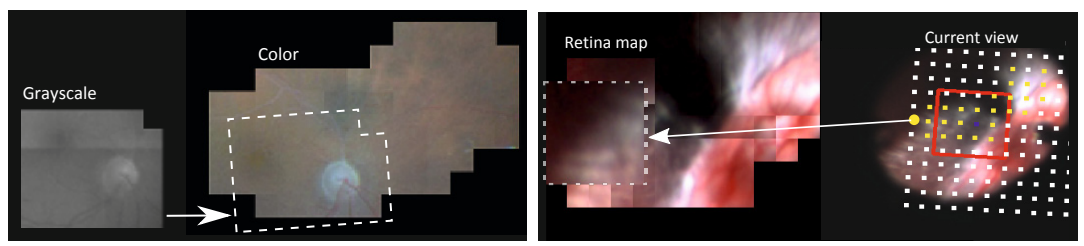

Fig. 5. (left) A considerably smaller retina map is obtained when tracking in gray-scale images. (right) Poor tracking quality measurements lead to the incorporation of wrong templates to the map in areas with little texture.
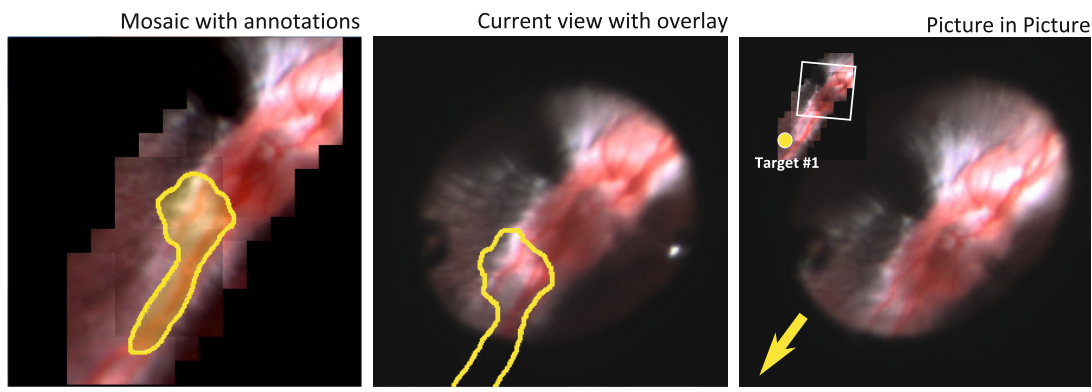

Fig. 6. Annotations created by a mentor on the intra-operative mosaic can be overlayed on the novice surgeon view for assistance and guidance during surgery. The mosaic could also be displayed to the surgeon for facilitating the localization of surgical targets.

circumvent this problem, consistency checks can be implemented for verifying the similarity between a given patch and its surrounding neighbors.

\section{Applications}

The proposed hybrid tracking and mosaicking method can be applied in a variety of scenarios. The most natural extension would be the creation of a photo realistic retina mosaic, taking advantage of the overlap between stored templates. The proposed system could also be used in an augmented reality scenario for tele-mentoring. Through intra-operative video overlay, a mentor could guide a novice surgeon by indicating points of interest on the retina, demonstrate surgical gestures or even create virtual fixtures in a robotic assisted scenario (see Figure 6(left-middle)). Similar to [4, the proposed tracking and mosaicking method can also be used for intra-operative guidance, facilitating the localization and identification of surgical targets as illustrated in Figure 6 (right). Videos illustrating these capabilities can be found in the supplementary materials.

\section{Conclusion}

In this paper we propose a hybrid tracking and mosaicking method for view expansion and surgical guidance during retinal surgery. The system is a combi- 
nation of direct and feature-based tracking methods. A novel extension for direct visual tracking using a robust similarity measure named SCV in color images is proposed. Several experiments conducted on phantom, in vivo rabbit and human images illustrate the ability of the method to cope with the challenging retinal surgery scenario. Furthermore, applications of the proposed method for tele-mentoring and intra-operative guidance are demonstrated.

Acknowledgements. This research was supported in part by NIH BRP grant 1 R01 EB 007969 and in part by Johns Hopkins University internal funds. Other equipment and systems infrastructure support were developed within the CISST ERC under NSF grant EEC9731748.

\section{References}

1. Mitchell, B., Koo, J., Iordachita, I., Kazanzides, P., Kapoor, A., Handa, J., Taylor, R., Hager, G.: Development and application of a new steady-hand manipulator for retinal surgery. In: ICRA, Rome, Italy, pp. 623-629 (2007)

2. Bergeles, C., Kummer, M.P., Kratochvil, B.E., Framme, C., Nelson, B.J.: Steerable Intravitreal Inserts for Drug Delivery: In Vitro and Ex Vivo Mobility Experiments. In: Fichtinger, G., Martel, A., Peters, T. (eds.) MICCAI 2011, Part I. LNCS, vol. 6891, pp. 33-40. Springer, Heidelberg (2011)

3. Balicki, M., Han, J., Iordachita, I., Gehlbach, P., Handa, J., Taylor, R., Kang, J.: Single Fiber Optical Coherence Tomography Microsurgical Instruments for Computer and Robot-Assisted Retinal Surgery. In: Yang, G.-Z., Hawkes, D., Rueckert, D., Noble, A., Taylor, C. (eds.) MICCAI 2009, Part I. LNCS, vol. 5761, pp. 108115. Springer, Heidelberg (2009)

4. Fleming, I., Voros, S., Vagvolgyi, B., Pezzementi, Z., Handa, J., Taylor, R., Hager, G.: Intraoperative visualization of anatomical targets in retinal surgery. In: IEEE Workshop on Applications of Computer Vision (WACV 2008), pp. 1-6 (2008)

5. Seshamani, S., Lau, W., Hager, G.: Real-Time Endoscopic Mosaicking. In: Larsen, R., Nielsen, M., Sporring, J. (eds.) MICCAI 2006, Part I. LNCS, vol. 4190, pp. 355-363. Springer, Heidelberg (2006)

6. Totz, J., Mountney, P., Stoyanov, D., Yang, G.-Z.: Dense Surface Reconstruction for Enhanced Navigation in MIS. In: Fichtinger, G., Martel, A., Peters, T. (eds.) MICCAI 2011, Part I. LNCS, vol. 6891, pp. 89-96. Springer, Heidelberg (2011)

7. Hu, M., Penney, G., Rueckert, D., Edwards, P., Bello, F., Figl, M., Casula, R., Cen, Y., Liu, J., Miao, Z., Hawkes, D.: A Robust Mosaicing Method with SuperResolution for Optical Medical Images. In: Liao, H., Eddie Edwards, P.J., Pan, X., Fan, Y., Yang, G.-Z. (eds.) MIAR 2010. LNCS, vol. 6326, pp. 373-382. Springer, Heidelberg (2010)

8. Broehan, A.M., Rudolph, T., Amstutz, C., Kowal, J.: Real-time multimodal retinal image registration for computed-assisted laser photocoagulation system. IEEE Transactions on Biomedical Engineering (TBME) 58(10), 2816-2824 (2011)

9. Silveira, G., Malis, E., Rives, P.: An efficient direct approach to visual SLAM. IEEE Transactions on Robotics 24(5), 969-979 (2008)

10. Richa, R., Sznitman, R., Taylor, R., Hager, G.: Visual tracking using the sum of conditional variance. In: IROS, San Francisco, USA, pp. 2953-2958 (2011)

11. Stewart, C., Tsai, L., Roysam, B.: The dual-bootstrap iterative closest point algorithm with application to retinal image registration. IEEE Transactions on Pattern Analysis and Machine Intelligence (PAMI) 22(1), 1379-1394 (2003) 